\title{
Resveratrol protects against oxidative stress by activating the Keap-1/Nrf2 antioxidant defense system in obese-asthmatic rats
}

\author{
XIAO-NAN LI ${ }^{1}$, LU-YI MA ${ }^{1}$, HONG JI ${ }^{1}$, YUAN-HUA QIN ${ }^{2}$, SHAN-SHAN JIN ${ }^{1}$ and LI-XIN XU ${ }^{1}$ \\ ${ }^{1}$ Department of Pediatrics, The First Affiliated Hospital of Dalian Medical University, Dalian, \\ Liaoning 116011; ${ }^{2}$ Department of Parasitology, Dalian Medical University, Dalian, Liaoning 116044, P.R. China
}

Received October 10, 2017; Accepted February 2, 2018

DOI: $10.3892 /$ etm.2018.6747

\begin{abstract}
The aim of the present study was to investigate the potential mechanism underlying the anti-obesity-asthmatic effects of resveratrol (RSV) in a rat model of obese-asthma. Rat models of obesity and asthma were established using a high-fat diet and the administration of ovalbumin, respectively. Rats were divided into 7 different groups: A normal control, a normal obese, a normal asthma, a normal obese + asthma, a RSV obese, a RSV asthma and a RSV obese + asthma group. Body weight, Lee index, body fat and lung histopathological changes were evaluated. Serum lipid levels were evaluated using calorimetric methods. Levels of reactive oxygen species (ROS) were examined using enzyme-linked immunosorbent assays. Cellular antioxidant enzyme activities were measured using commercial kits. Levels of kelch-like ECH associated protein 1 (Keap-1) and nuclear factor erythroid 2-related factor 2 (Nrf2) was examined using western blot analysis. The results indicated that obese and asthma rat models were successfully established. It was also demonstrated that RSV decreased fasting blood glucose in obese, asthmatic and obese-asthmatic rats. RSV altered serum lipid levels; it significantly increased high density lipoprotein cholesterol levels and significantly decreased serum triglyceride, serum total cholesterol and very low density lipoprotein levels, compared with untreated obese, asthmatic and obese-asthmatic rats $(\mathrm{P}<0.05)$. ROS levels were significantly decreased in the RSV treatment group compared with obese, asthmatic and obese-asthmatic rats $(\mathrm{P}<0.05)$. RSV treatment significantly increased catalase, glutathione, glutathione peroxidase and total superoxide dismutase levels compared with untreated obese, asthmatic and obese-asthmatic rats $(\mathrm{P}<0.05)$. Furthermore, RSV treatment significantly downregulated Keap-1 and upregulated Nrf2 levels in the heart, lung and kidney tissues of rats compared with untreated
\end{abstract}

Correspondence to: Professor Li-Xin Xu, Department of Pediatrics, The First Affiliated Hospital of Dalian Medical University, 222 Zhongshan Road, Dalian, Liaoning 116011, P.R. China E-mail: dllixiaonan@hainan.net

Key words: obese, asthma, resveratrol, kelch-like ECH associated protein 1/nuclear factor erythroid 2-related factor 2, oxidative stress controls. Therefore, the results demonstrate that RSV protects against oxidative stress by activating the Keap-1/Nrf2 antioxidant defense system in obese-asthmatic rat models.

\section{Introduction}

Obesity is becoming a serious global health problem; rates of obesity have doubled over the past 20-years $(1,2)$. Obesity increases the risk of certain diseases, including cardiovascular disease, diabetes, kidney injury, different types of cancer and various respiratory diseases (3). Epidemiological studies have identified a correlation between obesity and asthma $(4,5)$. Obesity markedly increases the risk of asthma occurring, increases the severity of asthma and decreases lung function (6). The results of animal studies have also indicated an association between obesity and asthma, and have demonstrated that obese and asthmatic animals asthmatic do not fully respond to therapy with the standard drugs used to treat asthma $(7,8)$. Therefore, it is important to develop a drug that inhibits the accumulation of blood fat in order to effectively treat obese patients with asthma.

Previous studies have demonstrated that obesity affects lung tissue; for example, in obesity-associated asthma, obesity induces inflammatory changes, including chronic systemic inflammation and increases levels of leukocytes and pro-inflammatory cytokines (9-11). Obesity is associated with increased oxidative stress and systemic inflammation; asthma is also associated with the increased expression of malondialdehyde, increased levels of reactive oxygen species (ROS) in membrane phospholipids and increased levels of oxidative stress $(12,13)$. Oxidative stress is characterized by increased ROS levels and the decreased production of antioxidants; these then induce various pathological changes that have critical implications in asthma. Therefore, the present study examined changes in levels of various oxidative biomarkers, including ROS, catalase (CAT), glutathione (14), glutathione peroxidase (GSH-Px) and total superoxide dismutase (T-SOD) in a rat model of obesity-asthma.

It has been suggested that the kelch-like ECH associated protein 1/nuclear factor erythroid 2-related factor 2 (Keap-1/Nrf-2) antioxidant defense system acts to decrease oxidative stress $(15,16)$. However, to the best of our knowledge, it has not yet been demonstrated whether the Keap-1/Nrf2 antioxidant defense system exhibits antioxidative activity 
in animals with obese-asthma. Therefore, the present study examined the association between the Keap-1/Nrf2 signaling pathway and oxidative stress-associated molecules in a rat model of obesity-asthma.

Resveratrol (RSV) is a type of non-flavonoid polyphenol that is naturally produced by numerous plants (17). It has been suggested that RSV may prevent or treat a wide range of disorders, including different types of cancer, ischemic diseases and cardiovascular disease (18). It has been demonstrated that the RSV may prevent obesity and help to regulate blood glucose levels in patients with diabetes (19). However, the mechanism by which RSV protects against obesity and regulates blood glucose levels remains unknown. Therefore, the present study aimed to investigate the potential mechanism by which RSV acts to protect against obesity-asthma in a rat model of obese-asthma.

\section{Materials and methods}

Animals. A total of 42 adult male Sprague-Dawley weighing 120-130 g were purchased from Beijing Hua Fukang Biotechnology Co., Ltd. (Beijing China). The rats were housed in cages, with $2-3$ rats/cage, at a temperature of $25 \pm 2^{\circ} \mathrm{C}$ and humidity of $50 \pm 5 \%$. The rats were kept in a $12 \mathrm{~h} \mathrm{light/dark}$ cycle and had free access to food and water. The rats in different groups were housed in separate cages. The present study was performed following the guidelines of the Care and Use of Laboratory Animals of National Institute of Health (20) and was approved by the Ethics Committee of Dalian Medical University (Dalian, China).

Establishment of the obese-asthmatic model and rat grouping. The 42 rats were randomly divided into the following 7 groups (all $n=6$ ): A normal control group, a normal asthma group, a normal obese group, an obese + asthmatic group, a RSV asthma group, a RSV obese group and a RSV obese + asthma group.

The normal control group was fed a normal diet for 6 weeks. The normal obese group was fed with high-fat diet for 6 weeks. Rats in the normal asthma group received an intraperitoneal injection of ovalbumin (OVA) suspension ( $0.5 \mathrm{ml} / \mathrm{rat})$, formed by dissolving $1 \mathrm{mg}$ OVA (Sigma-Aldrich; Merck KGaA, Darmstadt, Germany) and $20 \mu \mathrm{g}$ calmogastrin (Sigma-Aldrich; Merck KGaA) into $0.5 \mathrm{ml} 0.9 \%$ normal saline, on the first and eighth day following 6 weeks of a normal diet. On the fifth day, $10 \mathrm{~g} / \mathrm{l}$ OVA suspension $(10 \mathrm{ml})$ was administrated to the normal asthma group rats using the atomization excitation method for $30 \mathrm{~min}$, for three times each week. Rats in the asthma groups received OVA over a 2 -week period. Rats in the obese + asthma group were fed a high-fat diet for 6 weeks and received OVA in the same manner as the normal asthma group, following a previously described protocol (21). Rats in the RSV asthma, RSV obese and RSV obese + asthma groups were fed either a normal or a high-fat diet for 6 weeks. Rats in the RSV and RSV obese + asthma groups received OVA in the same manner as the normal asthma and obese + asthma groups. All rats in these groups received a supplement of $0.03 \%$ RSV (Nanjing Oddfoni Biol. Tech. Co. Ltd., Nanjing, China) in their diet (21).

Body weight evaluation. Rats were weighed every day. The rats were weighed prior to anesthetization. All rats were anesthetized using ketamine $(50 \mathrm{mg} / \mathrm{kg})$ and xylazine $(10 \mathrm{mg} / \mathrm{kg})$. Changes in body weight $(\%)$, the Lee index $(\mathrm{mg} / \mathrm{mm})$ and body fat percentage $(\%)$ were evaluated and calculated following previously published protocols $(22,23)$.

Lung histopathological evaluation in obese-asthma rat model. The rats were sacrificed by $100 \mathrm{mg} / \mathrm{kg}$ body weight intraperitoneal injection of thiopental (Shanghai New Asiatic Pharmaceutical Co. Ltd., China) and the lung, heart and kidneys were immediately removed for histopathological evaluation. Sacrifice was confirmed when rats stopped breathing and their hearts ceased beating. The tissues were fixed using $4 \%$ formaldehyde (Beyotime Institute of Biotechnology, Haimen, China) in PBS for $15 \mathrm{~min}$ at room temperature. The endogenous peroxidase was inactivated by $3 \%$ hydrogen peroxide for $5 \mathrm{~min}$ at room temperature. The tissues were cut into of $4-\mu \mathrm{m}$-thick sections. The sections were stained with haematoxylin-eosin at room temperature for $15 \mathrm{~min}$. Lung tissues were evaluated using a light microscope. Pathological changes in the lung tissues, including airway smooth muscle hyperplasia, emphysema and edematous changes, were recorded and calculated according following a previously published protocol (21). Pathological grades were defined using the following system (21): Grade 0, no pathological changes; grade 1, patchy pathological changes; grade 2 , local pathological changes; grade 3, scatter pathological changes and grade 4 , severe pathological changes in most parts of the lung tissue.

Blood glucose and serum lipid evaluation. All rats were fasted overnight for $\geq 8 \mathrm{~h}$ and $2 \mathrm{ml}$ blood was taken from the tail vein in the morning. Blood glucose was measured using an On-Call glucose analyzer (ACON Biotech Hangzhou Co., Ltd., Hangzhou, China). Levels of fasting serum lipids, including high density lipoprotein cholesterol (HDL-C; cat. no. A112-1), serum triglyceride (TG; cat. no. A110-1), serum total cholesterol (TC; cat. no. A111-1) and very low density lipoprotein (vLDL; cat. no. H249) levels were evaluated using ELISA with the HDL-C, TG, TC, vLDL test kits (Nanjing Jiancheng Bioengineering Institute, Nanjing, China), respectively. The fasting serum lipids were examined using an ELISA reader (ELX800; BioTek Instruments, Inc., Winooski, VT, USA). Levels of serum lipids were calculated using the Friedewald Equation (24).

ROS determination. Rat tissues (heart, lung and kidney) were washed with PBS in ice to remove blood and necrotic tissue. Fibrosis, fat and blood vessels were also removed. Subsequently, tissues were sliced into $1-\mathrm{mm}^{3}$ sections and these slices were ground to obtain the cell suspension. Cell suspensions were centrifuged at a speed of $500 \mathrm{x}$ g for $10 \mathrm{~min}$ at room temperature and supernatants were then removed. ROS levels in the heart, tissue and lungs of rats from each group were measured using an enzyme-linked immunosorbent assay kit (cat. no. 88-5930; Beijing Leagene Biotech Co., Ltd., Beijing, China) following the manufacturer's protocol. Obtained cells were resuspended in PBS and the cell density was adjusted to $10^{6} / \mathrm{sample}$. Cells were then incubated with $10 \mu \mathrm{M}$ dichloro-dihydro-fluorescein diacetate at $37^{\circ} \mathrm{C}$ for $45 \mathrm{~min}$, centrifuged at $1,000 \mathrm{x} \mathrm{g}$ for $10 \mathrm{~min}$ at $37^{\circ} \mathrm{C}$ and 
cells were then collected. Cell fluorescence was measured at $500 / 525 \mathrm{~nm}$ using a microplate reader (Synergy ${ }^{\mathrm{TM}}$ H4; BioTek Instruments, Inc.).

Evaluation of cellular antioxidant enzyme activities. The activities of cellular antioxidant enzymes were evaluated using a CAT assay kit (cat. no. TO1070-100T), a GSH assay kit (cat. no. TO1031-100T) (both Beijing Leagene Biotech Co., Ltd.), a T-SOD assay kit (cat. no. A001-1-1) and a GSH-PX assay kit (cat. no. A005) (both Nanjing Jiancheng Bioengineering Institute) following the manufacturer's protocol. Tissues were treated using the aforementioned processes used to determine ROS levels; supernatants were removed and pellets were collected post-centrifugation. Pellets were then lysed using $0.5 \%$ trypsin at $37^{\circ} \mathrm{C}$ for $20 \mathrm{~min}$. Lysed cells were then centrifuged at $10,000 \mathrm{x} \mathrm{g}$ at room temperature for $10 \mathrm{~min}$ and supernatants were then collected. CAT activity was evaluated at $562 \mathrm{~nm}$ and calculated according to the $\mathrm{H}_{2} \mathrm{O}_{2}$ decomposition rate at $405 \mathrm{~nm}$. GSH activity was evaluated at $420 \mathrm{~nm}$ and calculated using the GSH calibration curve. T-SOD activity was evaluated at $560 \mathrm{~nm}$ and was determined according to its ability to suppress the oxidation of hydroxylamine by the superoxide anion from the xanthine oxidase system. GSH-Px activity was evaluated at $412 \mathrm{~nm}$ according to the oxidation rate of decreased glutathione to the oxidized glutathione by $\mathrm{H}_{2} \mathrm{O}_{2}$ under GSH-Px catalysis using a microplate reader.

Western blot assay. Tissues were lysed by using radioimmuniprecipitation assay buffer $(1 \mathrm{ml} / 100 \mathrm{mg})$ at $0^{\circ} \mathrm{C}$ and were then centrifuged at $10,000 \times \mathrm{g}$ for $5 \mathrm{~min}$ at $4^{\circ} \mathrm{C}$. The concentration of the obtained proteins was determined by using bicinchoninic acid protein assay kit (cat. no. 23227; Thermo Fisher Scientific, Inc., Waltham, MA, USA). The obtained supernatant $(0.2 \mu \mathrm{g}$ protein per lane) then underwent 15\% SDS-PAGE (GE Healthcare, Little Chalfont, UK) and was then electrotransferred onto PVDF membranes (Bio-Rad Laboratories, Inc., Hercules, CA, USA). PVDF membranes were blocked using 5\% bovine serum albumin (cat. no. 15260-037; Gibco; Thermo Fisher Scientific, Inc.) in PBS (supplemented with $0.05 \%$ Tween-20 solution) at a $\mathrm{pH}$ of 7.5 for $2 \mathrm{~h}$ at room temperature. Subsequently, membranes were incubated with rabbit anti-rat Keap-1 polyclonal antibody (1:3,000; cat. no. ab139729), rabbit anti-rat Nrf2 monoclonal antibody (1:3,000; cat. no. ab181602) and rabbit anti-rat GAPDH polyclonal antibody $(1: 2,000$; cat. no. ab9485) (all Abcam, Cambridge, MA, USA) at room temperature for $2 \mathrm{~h}$. Membranes were washed using PBST and subsequently incubated with horseradish peroxidase-conjugated goat anti-rabbit immunoglobulin G (1:2,000; cat. no. A0545; Sigma-Aldrich; Merck KGaA, Darmstadt, Germany). Reactive protein signals were visualized using an enhanced chemiluminescence kit (Thermo Fisher Scientific, Inc.). Finally, gel images were scanned and analyzed using a GDS8000 image scanning system (UVP, LLC, Upland, CA, USA).

Statistical analysis. All data were assessed using SPSS software 20.0 (IBM Corp, Armonk, NY, USA) and presented as the mean \pm standard deviation. All data were obtained from at least six independent experiments or tests. A Student's t test was used to determine whether differences between two groups were significant. One-way analysis of variance followed by
Tukey's post hoc test was used to compare measurement data among multiple groups. $\mathrm{P}<0.05$ was determined to indicate a statistically significant difference.

\section{Results}

Successful establishment of obese-asthma rat models. To confirm the establishment of the obese, asthma and obese-asthma rat models, changes in body weight, the Lee index and body fat percentage were assessed to determine the establishment of the obese models. Airway smooth muscle hyperplasia, emphysema and edematous changes, were assessed to determine the establishment of the asthmatic models. The results demonstrated that all obese indices, including body weight (Fig. 1A), Lee index values (Fig. 1B) and body fat percentage (Fig. 1C) were significantly increased in the obese-asthma and normal obese groups compared with the normal control group (all $\mathrm{P}<0.05$ ). Furthermore, all asthma associated indices, including emphysema (Fig. 1D), airway smooth muscle hyperplasia (Fig. 1E) and edematous changes (Fig. 1F), were all significantly increased in the normal- and obese-asthma normal groups compared with the normal control group (all $\mathrm{P}<0.05$ ).

$R S V$ decreases fasting blood glucose levels. To evaluate the effects of RSV on blood glucose levels in obese-asthmatic rats, fasting blood glucose levels were examined. The results indicated that fasting blood glucose levels in the RSV asthma group were significantly decreased compared with the normal asthma group ( $\mathrm{P}<0.05$; Fig. $2 \mathrm{~A})$ and that fasting blood glucose levels in the RSV obese group were also significantly decreased compared with the normal obese group $(\mathrm{P}<0.05)$. Furthermore, the fasting blood glucose levels were significantly reduced in the RSV obese + asthma group compared with the normal obese + asthma group ( $\mathrm{P}<0.05$; Fig. $2 \mathrm{~A})$. There were no significant differences in fasting blood glucose levels between the normal obese and asthma groups (Fig. 2A).

$R S V$ improves serum lipid levels. To investigate the effects of RSV on serum lipids, HDL-C, TG, TC and vLDL levels were evaluated in the present study. The results demonstrated that HDL-C levels in the RSV asthma group were significantly increased compared with the normal asthma group $(\mathrm{P}<0.05$; Fig. 2B) and were also significantly increased in the RSV obese group compared with the normal obese group $(\mathrm{P}<0.05)$. Furthermore, HDL-C levels were significantly increased in the RSV obese + asthma group compared with the normal obese + asthma group ( $\mathrm{P}<0.05$; Fig. 2B). By contrast, TG (Fig. 2C), TC (Fig. 2D) and vLDL (Fig. 2E) levels were significantly decreased in the RSV asthma group compared with the normal asthma group, in the RSV obese group compared with the normal obese group and in the RSV obese + asthma group compared with the normal obese + asthma group (all $\mathrm{P}<0.05$ ). There were no significant differences in HDL-C, TG, TC and vLDL levels between the normal obese and normal asthma groups (Fig. 2).

$R S V$ inhibits ROS production. ROS levels may reflect oxidative status; therefore, ROS levels were measured in the heart, lung and kidney tissues of obese-asthmatic rats. The results indicated that ROS levels in the heart tissue were significantly decreased in the RSV asthma, RSV obese and RSV 

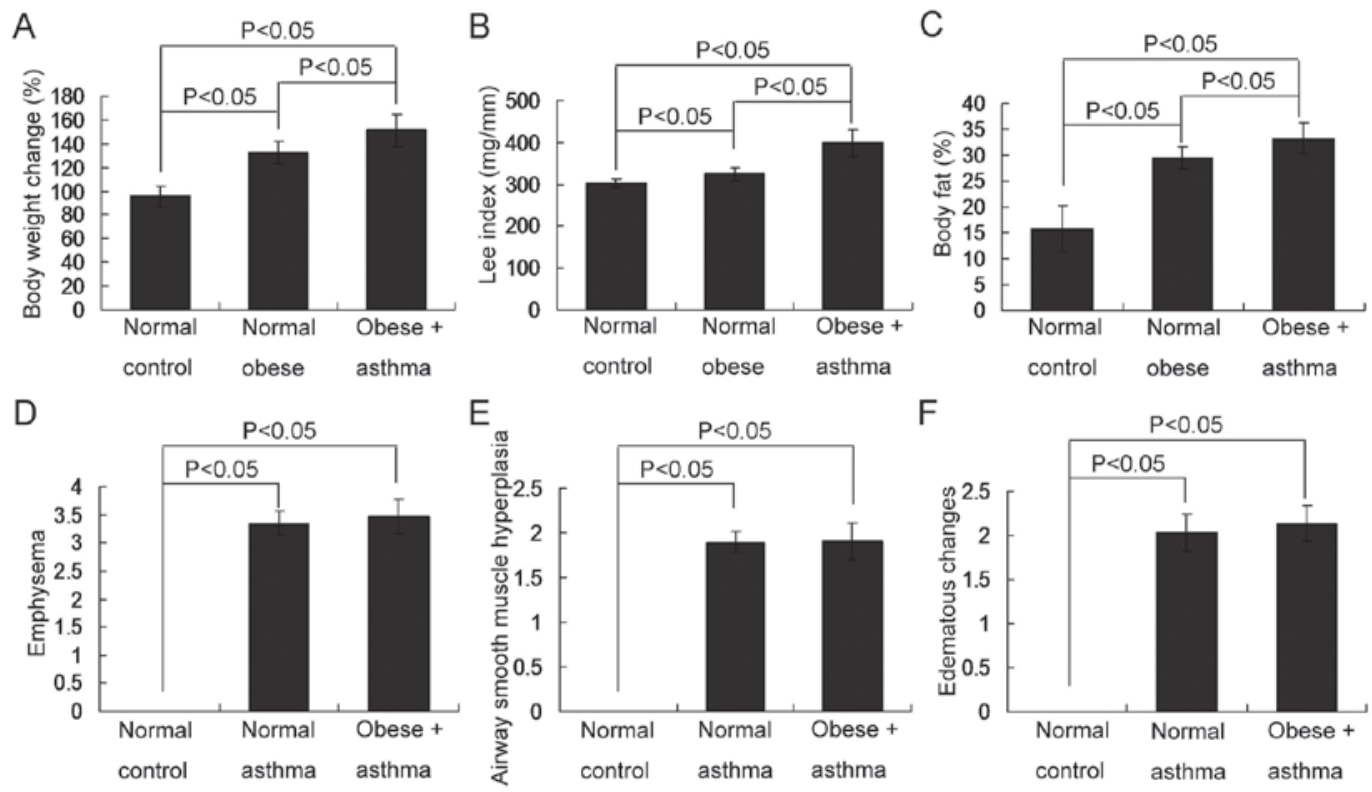

Figure 1. Changes in the obese associated and asthma associated indices in obese, asthma and obese-asthmatic rats. (A) Changes in rat body weights; (B) changes in the Lee index; (C) changes in body fat percentage; determining the presence of (D) emphysema; (E) airway smooth muscle hyperplasia and (F) edematous changes. All data are presented as the mean \pm standard deviation and were obtained from at least six independent experiments or tests.
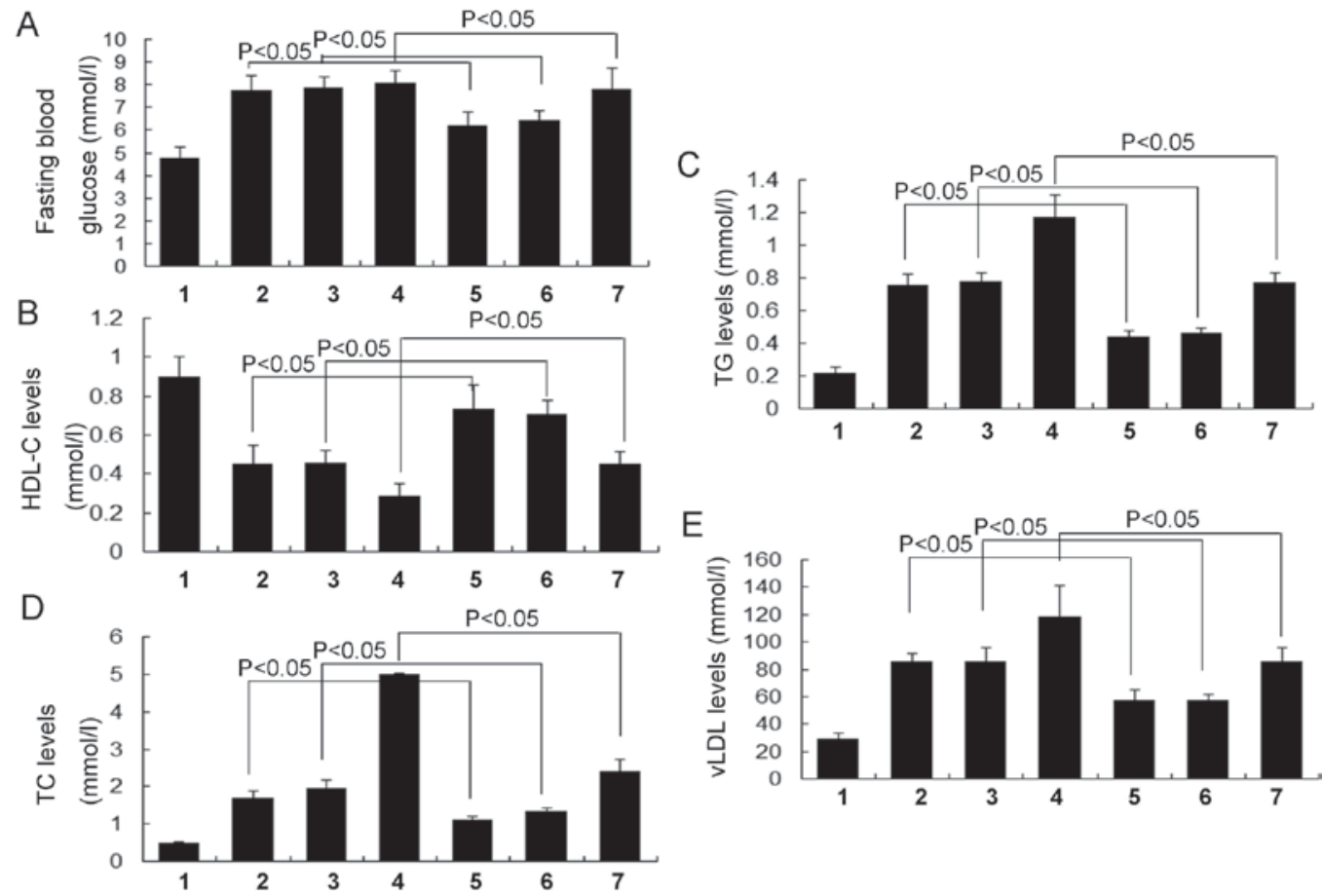

Figure 2. Evaluation of fasting blood glucose and serum lipid levels. (A) Fasting blood glucose, (B) HDL-C, (C) TG, (D) TC and (E) vLDL levels. All data are presented as the mean \pm standard deviation and were obtained from at least six independent experiments or tests. 1, Normal control group; 2, Normal obese group; 3, Normal asthma group; 4, Obese + asthma group; 5, RSV obese group; 6, RSV asthma group; 7, RSV obese + asthma group; RSV, resveratrol; HDL-C, high density lipoprotein cholesterol; TG, serum triglyceride; TC, serum total cholesterol; vLDL, very low density lipoprotein.

obese + asthma groups compared with the normal asthma, normal obese and normal obese + asthma group, respectively (all $\mathrm{P}<0.05$; Fig. 3A). ROS levels in the lung (Fig. 3B) and kidney tissue (Fig. 3C) were also significantly decreased in each of the groups that received RSV treatment, compared with their respective controls $(\mathrm{P}<0.05)$. Furthermore, there were no significant differences in ROS levels in the lung, kidney and heart tissues between the normal obese and asthma groups (Fig. 3).

$R S V$ enhances cellular antioxidant enzyme activities. In the present study, levels of antioxidant enzymes including CAT, GSH, GSH-Px and T-SOD, in the heart, lung and kidney tissue of rats were analyzed using commercial kits. The results 
A

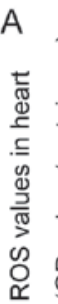

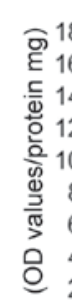

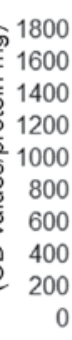

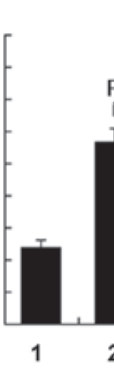
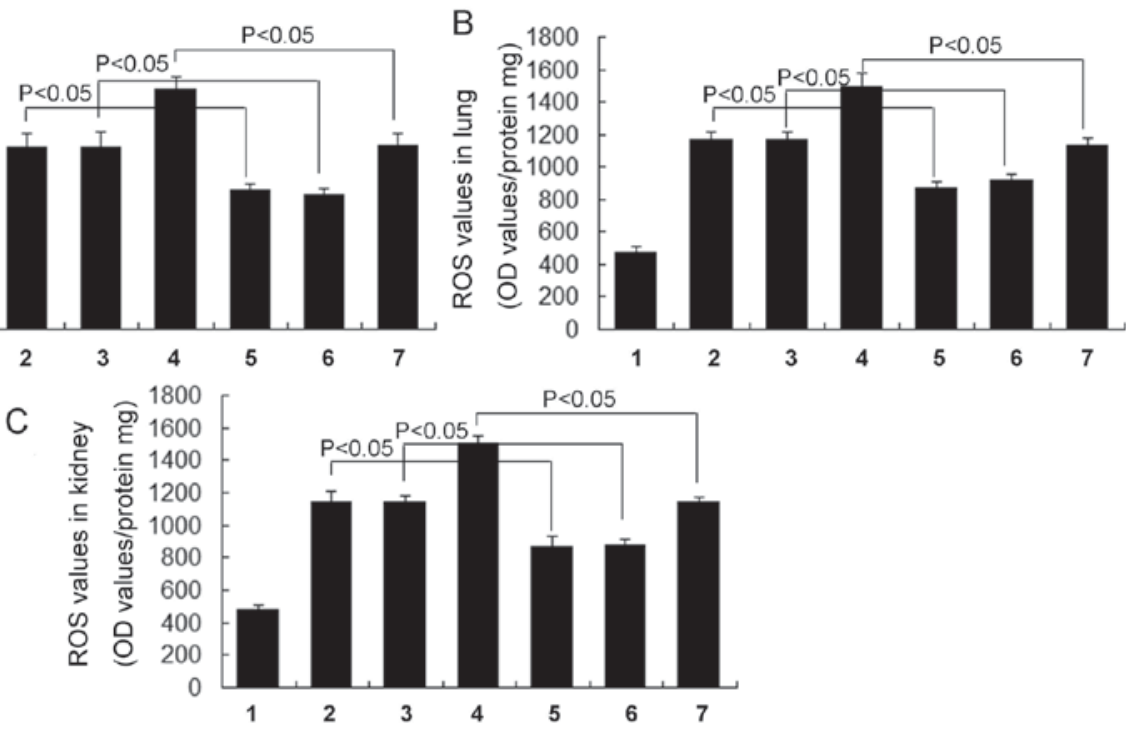

Figure 3. ROS levels in different tissues. ROS levels in the (A) heart, (B) lung and (C) kidney tissue. All data are presented as the mean \pm standard deviation and were obtained from at least six independent experiments or tests. 1, Normal control group; 2, Normal obese group; 3, Normal asthma group; 4, Obese + asthma group; 5, RSV obese group; 6, RSV asthma group; 7, RSV obese + asthma group; ROS, reactive oxygen species; RSV, resveratrol; OD, optical density.

demonstrated that RSV treatment significantly increased CAT levels in all groups compared with their respective controls (all $\mathrm{P}<0.05)$. This was the case in the heart (Fig. 4A), lung (Fig. 4B) and kidney tissues (Fig. 4C). There were no significant differences in CAT levels between the normal obese and asthma groups in any of the tissues assessed (Fig. 4).

GSH (Fig. 5), GSH-Px (Fig. 6) and T-SOD levels (Fig. 7) were all significantly increased in each of the RSV treatment groups (the RSV asthma, RSV obese and RSV obese + asthma groups) compared with their respective controls (the normal asthma, normal obese and normal obese + asthma groups, respectively) in the heart, lung and kidney tissues (all $\mathrm{P}<0.05$ ). There were no significant differences in GSH (Fig. 5), GSH-Px (Fig. 6) and T-SOD levels (Fig. 7) in the lung, kidney and heart tissues between the normal obese and asthma groups.

$R S V$ downregulates Keap-1 expression. In the present study, the Keap-1/Nrf-2 antioxidant defense system molecule, Keap-1, was assessed via western blotting. The results indicated that Keap-1 expression in the RSV asthma group was significantly decreased compared with the normal asthma group in heart tissue ( $\mathrm{P}<0.05$; Fig. 8A). Keap-1 expression was also significantly decreased in the RSV obese group compared with the normal obese group $(\mathrm{P}<0.05$; Fig. 8A). Furthermore, Keap-1 expression was significantly decreased in the RSV obese + asthma group compared with the normal obese + asthma group $(\mathrm{P}<0.05$; Fig. $8 \mathrm{~A})$.

The expression of Keap-1 in the lung and kidney tissues was also examined. The results demonstrated that RSV treatment significantly decreased Keap-1 expression in the lung (Fig. 8B) and kidney tissue (Fig. 8C; all $\mathrm{P}<0.05$ ).

There were no significant differences in Keap-1 expression in any of the tissues between the normal obese and asthma groups in any of the tissues assessed (Fig. 8).

$R S V$ upregulates Nrf2 expression. Another Keap-1/Nrf2 antioxidant defense system molecule, Nrf2, was also examined in the present study. The results demonstrated that Nrf2 expression in the RSV asthma and RSV obese groups was significantly increased compared with the normal asthma and normal obese groups, respectively, in the heart tissue $(\mathrm{P}<0.05$; Fig. 8A). Furthermore, Nrf2 expression was significantly increased in the RSV obese + asthma group compared with the normal obese + asthma group $(\mathrm{P}<0.05$; Fig. 8A).

Nrf2 expression in the lung and kidney tissues was also examined. The results indicated that the RSV treatment significantly increased Nrf2 expression in the lung (Fig. 8B) and kidney tissue (Fig. 8C) in all groups that received RSV treatment, compared with their respective controls (all $\mathrm{P}<0.05$ ).

There were no significant differences in Nrf-2 expression in the lung, kidney and heart tissue between the normal obese and asthma groups (Fig. 8).

\section{Discussion}

Obesity is considered to be a chronic and low-grade pro-inflammatory disease; however, it remains unknown whether this inflammation induces the development of asthma and the inflammation of airways in humans or animals $(23,25)$. Therefore, the current study administered a high-fat diet to rats in order to establish a model of obesity, used OVA to establish a model of asthma and assessed whether there is an association between ROS/antioxidant levels and obese-asthma.

The results of the present study indicated that rats with diet-induced obesity exhibited weight gain, increased Lee index values and fat percentages, which was consistent with the results of a previous study (26). Furthermore, rats with OVA-induced asthma exhibited airway smooth muscle hyperplasia, emphysema and edematous changes, which were also consistent with the results of a previous study (27). These changes in obesity and asthma indices suggest that the obese, asthma and obese-asthmatic models were all established successfully, and therefore could be used in subsequent experiments. 

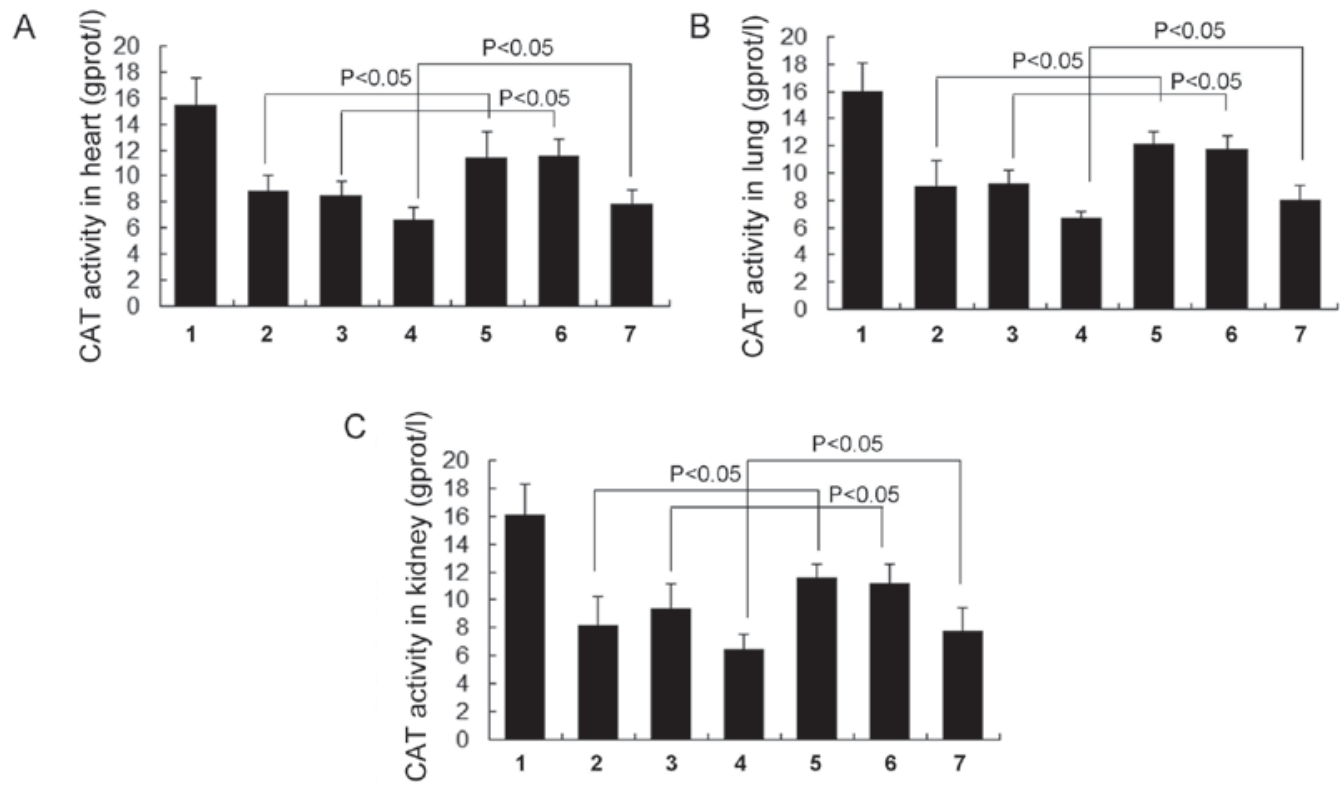

Figure 4. Activity of the cellular antioxidant enzyme CAT in different tissues. CAT activity in the (A) heart, (B) lung and (C) kidney tissue. All data are presented as the mean \pm standard deviation and were obtained from at least six independent experiments or tests. 1, Normal control group; 2, Normal obese group; 3, Normal asthma group; 4, Obese + asthma group; 5, RSV obese group; 6, RSV asthma group; 7, RSV obese + asthma group; CAT, catalase; RSV, resveratrol.
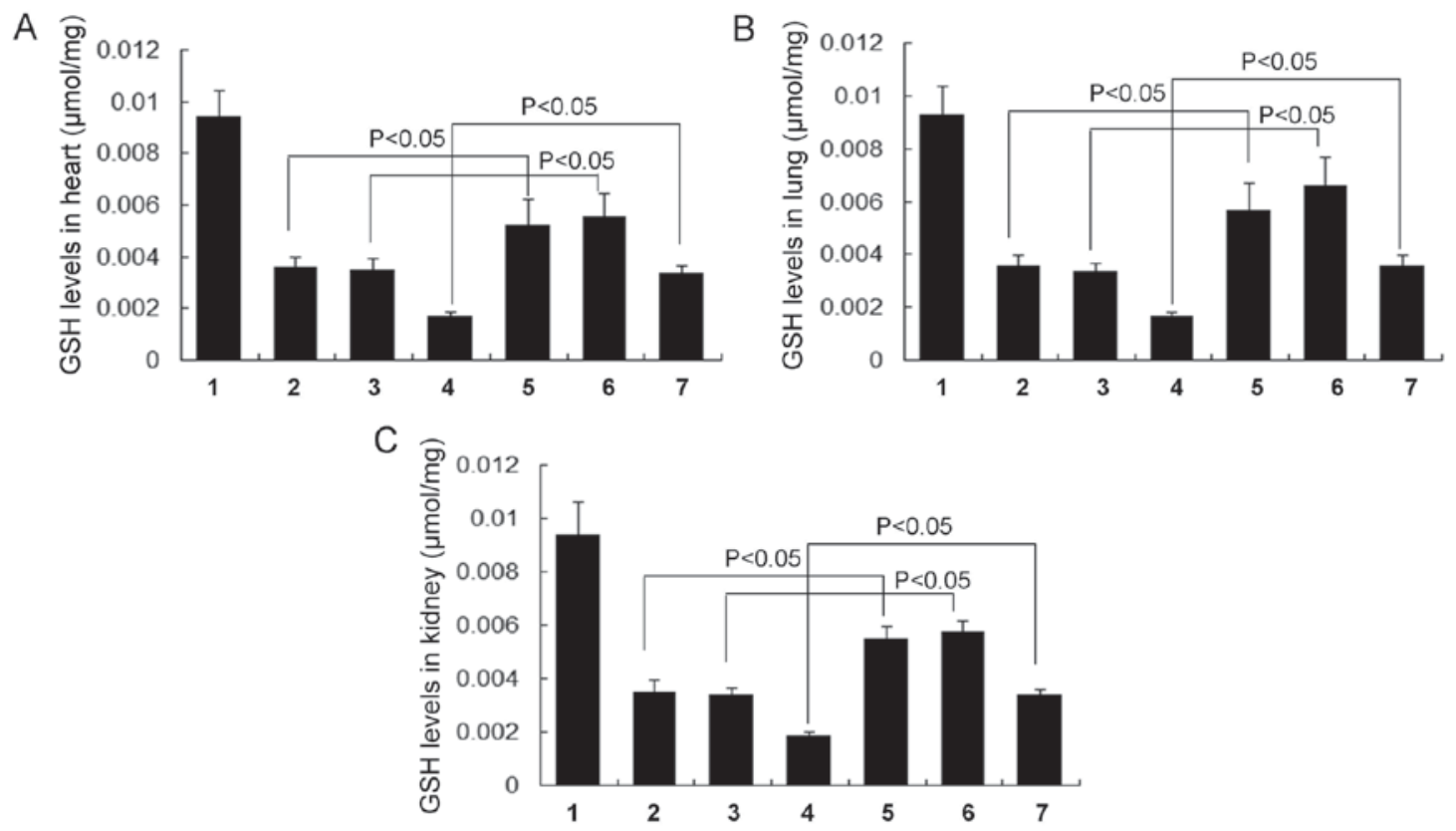

Figure 5. Levels of the cellular antioxidant enzyme GSH in different tissues. GSH levels in the (A) heart, (B) lung and (C) kidney tissue. All data are presented as the mean \pm standard deviation and were obtained from at least six independent experiments or tests. 1, Normal control group; 2, Normal obese group; 3, Normal asthma group; 4, Obese + asthma group; 5, RSV obese group; 6, RSV asthma group; 7, RSV obese + asthma group; RSV, resveratrol; GSH, glutathione.

Previous studies have investigated the effects of RSV in the treatment of various diseases, including Alzheimer's disease (28), heart disease (29) and Parkinson's disease (30). Studies have also investigated the potential of RSV to treat obesity-associated diseases, including non-alcoholic fatty liver disease (31) and the asthmatic disorders, such as allergic asthma (32). However, the specific mechanisms by which RSV induces therapeutic effects in such disorders remains unknown. Therefore, the current study investigated the anti-obese and anti-asthmatic effects of RSV and its associated mechanisms of action.

The results of the current study indicated that the products of diet-induced obesity, including HDL-C, TG, TC and vLDL, were significantly increased in obese and asthmatic rats compared with normal control rats. However, RSV significantly reversed the increase in HDL-C, TG, TC and vLDL that occurred in obese and asthmatic rats. These effects of RSV are consistent with the results observed in 
A

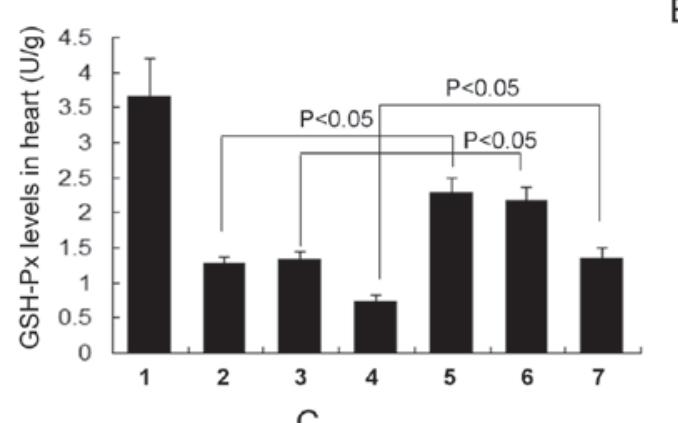

B

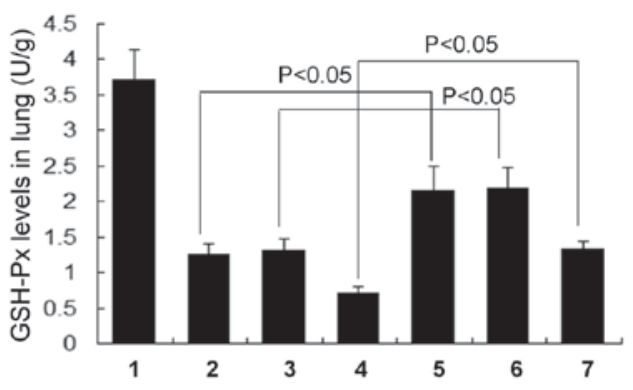

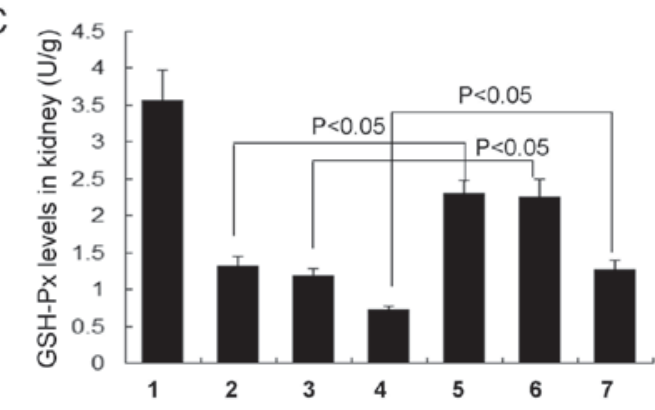

Figure 6. Levels of the cellular antioxidant enzyme GSH-Px in different tissues. GSH-Px levels in the (A) heart, (B) lung and (C) kidney tissue. All data are presented as the mean \pm standard deviation and were obtained from at least six independent experiments or tests. 1, Normal control group; 2, Normal obese group; 3, Normal asthma group; 4, Obese + asthma group; 5, RSV obese group; 6, RSV asthma group; 7, RSV obese + asthma group; RSV, resveratol; GSH-Px, glutathione peroxidase.

A

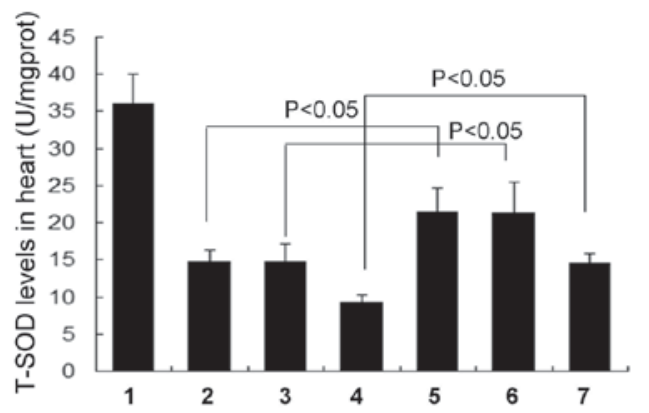

B

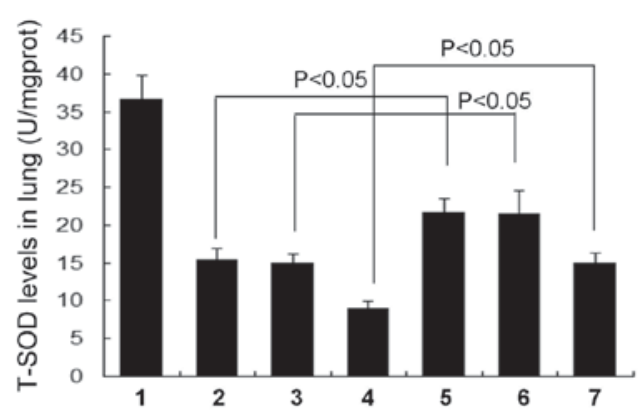

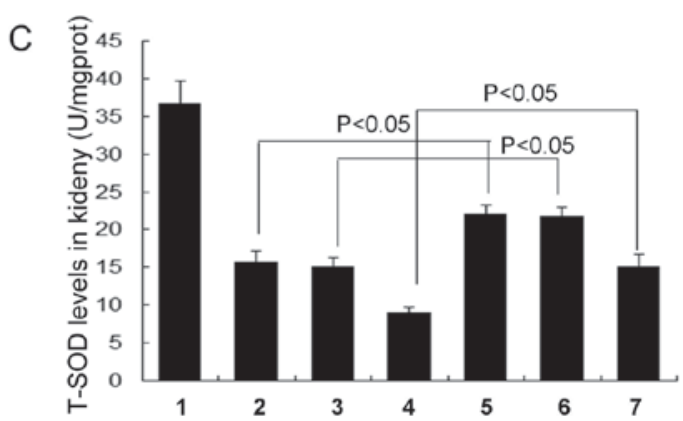

Figure 7. Levels of the cellular antioxidant enzyme T-SOD in different tissues. T-SOD levels in the (A) heart, (B) lung and (C) kidney tissue. All data are presented as the mean \pm standard deviation and were obtained from at least six independent experiments or tests. 1, Normal control group; 2, Normal obese group; 3, Normal asthma group; 4, Obese + asthma group; 5, RSV obese group; 6, RSV asthma group; 7, RSV obese + asthma group; RSV, resveratrol; T-SOD, total superoxide dismutase.

a previous study (33), which indicated that RSV improves lipid metabolism in patients with liver disease. Xie et al (34) also reported that RSV regulates lipid metabolism in hyperlipidemic mice. The effects of RSV on lipid metabolism suggest that it may be used to treat patients with hyperlipoidemia-associated disorders. However, obesity-associated parameters, including fasting blood glucose, HDL-C, TG, TC and vLDL levels in asthmatic rats were also enhanced compared with normal rats. These results regarding lipid-associated parameters in asthma are similar. The results of a previous study investigating patients with severe acute asthma indicated that they exhibited increased 

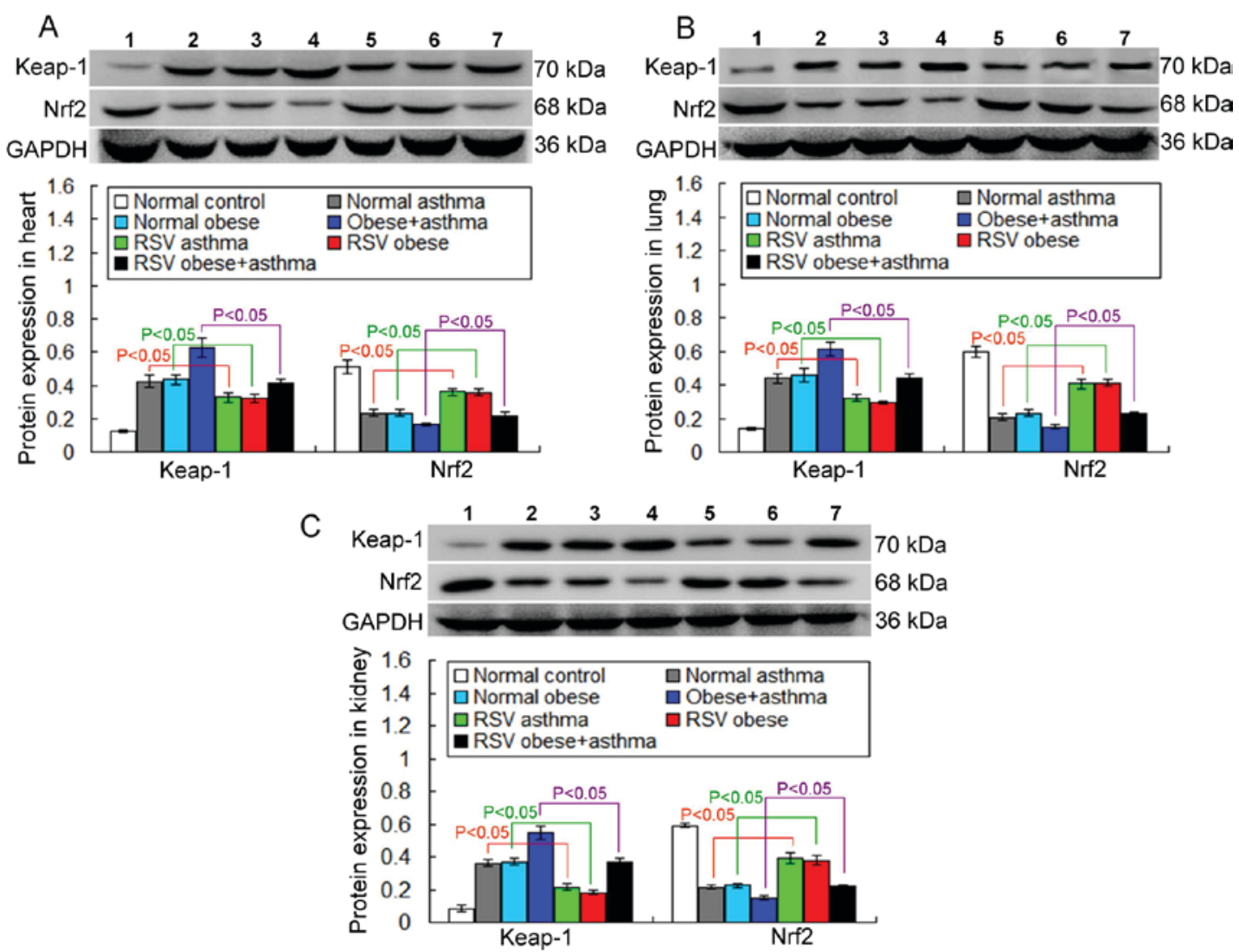

Figure 8. Keap-1 and Nrf2 expression in different tissues measured via western blot analysis. The expression of Keap-1 and Nrf2 in the (A) heart, (B) lung and (C) kidney tissue. All data are presented as the mean \pm standard deviation and were obtained from at least six independent experiments or tests. 1, Normal control group; 2, Normal obese group; 3, Normal asthma group; 4, Obese + asthma group; 5, RSV obese group; 6, RSV asthma group; 7, RSV obese + asthma group; RSV, resveratrol; Keap-1, kelch-like ECH associated protein 1; Nrf2, nuclear factor erythroid 2-related factor 2.

hyperglycemia and hyperlipemia (35). In the current study, a rat model of acute asthma was established by intraperitoneal administration of OVA suspension. It may be that the increased levels of HDL-C, TG, TC and vLDL observed in rats with asthma observed in the current study were caused by the acute administration of OVA suspension. Further studies are required to explain these changes in HDL-C, TG, TC and vLDL levels that occurred in the asthmatic rats included in the present study.

Oxidative stress has two main effects on intercellular physiological processes; it enhances ROS production and decreases levels of antioxidants (36). Increased oxidative stress in airways is considered to be a mechanism by which obesity enhances the severity of asthma $(13,37)$. Ochs-Balcom et al (38) also demonstrated that obese-asthmatic patients with obstructed airways experienced more severe asthma and severe oxidative stress. It was hypothesized that the imbalance between antioxidants and ROS may serve a critical role in the pathological process of obese-asthma. Therefore, the current study evaluated ROS and antioxidant (CAT, GSH, GSH-Px and T-SOD) levels in the heart, lung and kidney tissues of obese and/or asthmatic rats undergoing RSV treatment. The results indicated that RSV treatment significantly decreased levels of ROS in the heart, lung and kidney tissues of obese and asthmatic rats. Furthermore, RSV significantly increased CAT, GSH, GSH-Px and T-SOD levels in the heart, lung and kidney tissues of obese and asthmatic rats. The antioxidative effects of RSV determined in the current study were consistent with the results of a study by Guo et al (39), which indicated that RSV inhibits high glucose-triggered oxidative stress by suppressing the production of ROS. Chen et al (40) also reported that RSV protects hepatic cells against oxidative stress by enhancing SOD activity. Although Sood et al (41) indicated that oxidative stress in airways may act as a signaling pathway between obesity and asthma, the present study is the first to indicate the role of RSV in protecting against oxidative stress by decreasing ROS levels and increasing the expression of CAT, GSH-Px, GSH and T-SOD in obese-asthmatic rat models.

It has been demonstrated that the Keap-1/Nrf2 antioxidant defense system is an important cyto-protective mechanism against the oxidative stress associated with increased ROS and decreased antioxidant levels (42). Therefore, it was speculated that the Keap-1/Nrf2 antioxidant system may participate in the antioxidative stress processes of RSV in obese-asthmatic rat models. Key molecules of the Keap-1/Nrf2 signaling pathway, including Keap-1 and Nrf2, were assessed in the current study. The results illustrated that RSV significantly downregulated Keap-1 and upregulated Nrf2 levels in obese, asthmatic and obese-asthmatic rats. Wang et al (43) reported that the mitogen-activated protein kinase/Nrf2 signaling pathway is stimulated in rats with high-fat diet induced obesity. Previous studies also explored the role of Nrf2 and Keap-1 in the development of allergic, bronchial and childhood asthma $(44,45)$. However, few studies have investigated the association between the 
pathogenesis of obesity or asthma and the Keap-1/Nrf2 antioxidant defense system; to the best of our knowledge, the present study was the first to do so. Furthermore, the current study also indicated that RSV triggered the downregulation of Keap-1 and increased the expression of Nrf2 in the obese-asthmatic rats, which is consistent with the results of a previous study (46). It has also been demonstrated that RSV attenuates obesity-associated inflammation by activating the AMP-activated protein kinase and its signaling pathway (47). However, the present study indicated that RSV attenuates obesity-asthmatic associated oxidative stress via the Keap-1/Nrf2 antioxidant defense system. This suggests that RSV prevents high-fat-induced obese and OVA-induced asthma by activating the Keap-1/Nrf2 antioxidant defense system and may therefore be a promising therapeutic target for treating obesity-asthma.

The current study had a few limitations. Firstly, dynamic changes in Keap-1/Nrf2 expression were not evaluated. Future studies should evaluate the expression of Nrf2 in the nuclear fraction and Keap-1 in the cytosolic fraction, following a previously described protocol (48). Secondly, Nrf-2 and Keap-1 expression are not always associated with $\mathrm{Nrf}-2$ activation (46); therefore, to ascertain whether Nrf-2 is activated, the expression of Nrf-2 nuclear translocation and/or the Nrf-2 target gene should be determined. Thirdly, the association between asthma and obesity was not investigated in the current study; therefore, it could not be clarified whether asthma is caused by obesity. Finally, it was not confirmed in the current study whether RSV regulates the Keap-1/Nrf-2 antioxidant defense system.

In conclusion, the results of the present study demonstrated that RSV treatment decreases serum lipid levels, decreases ROS production and increases antioxidant enzyme activities in a rat model of obese-asthma. Furthermore, RSV treatment decreased Keap-1 and increased Nrf2 levels. Therefore, the results of the current study indicate that RSV protects against obese-asthma associated oxidative stress by activating the Keap-1/Nrf2 antioxidant defense system in obese-asthmatic rats.

\section{Acknowledgements}

Not applicable.

\section{Funding}

No funding was received.

\section{Availability of data and materials}

All data generated or analyzed during this study are included in this published article.

\section{Authors' contributions}

XNL, LYM, HJ, YHQ performed the ELISA assays and western blot analysis. XNL and SSJ analyzed the patient data. XNL and LXX wrote the manuscript and were the primary designers of the study. All authors read and approved the final manuscript.

\section{Ethics approval and consent to participate}

The present study was performed following the guidelines of the Care and Use of Laboratory Animals of National Institute of Health and was approved by the Ethics Committee of Dalian Medical University (Dalian, China).

\section{Patient consent for publication}

Not applicable.

\section{Competing interests}

The authors declare that they have no competing interests.

\section{References}

1. Li L, Wang G, Li N, Yu H, Si J and Wang J: Identification of key genes and pathways associated with obesity in children. Exp Ther Med 14: 1065-1073, 2017.

2. Shore SA and Fredberg JJ: Obesity, smooth muscle, and airway hyperresponsiveness. J Allergy Clin Immunol 115: 925-927, 2005.

3. McClean KM, Kee F, Young IS and Elborn JS: Obesity and the lung: 1. Epidemiology. Thorax 63: 649-654, 2008.

4. Beuther DA and Sutherland ER: Overweight, obesity, and incident asthma: A meta-analysis of prospective epidemiologic studies. Am J Respir Crit Care Med 175: 661-666, 2007.

5. Zhang S, Yang J, Zheng R, Jiang L, Wei Y and Liu G: VanWyk-grumbach syndrome in a male pediatric patient: A rare case report and literature review. Exp Ther Med 13: 1151-1154, 2017.

6. Leynaert B, Sunyer J, Garcia-Esteban R, Svanes C, Jarvis D, Cerveri I, Dratva J, Gislason T, Heinrich J, Janson C, et al: Gender differences in prevalence, diagnosis and incidence of allergic and non-allergic asthma: A population-based cohort. Thorax 67: 625-631, 2012.

7. Shore SA, Schwartzman IN, Mellema MS, Flynt L, Imrich A and Johnston RA: Effect of leptin on allergic airway responses in mice. J Allergy Clin Immunol 115: 103-109, 2005.

8. Dixon AE and Poynter ME: Mechanisms of asthma in obesity. pleiotropic aspects of obesity produce distinct asthma phenotypes. Am J Respir Cell Mol Biol 54: 601-608, 2016.

9. Hwang SJ, Kim JH, Shim JW, Kim DS, Jung HL, Park MS Lee WY, Kim SY and Shim JY: Peroxisome proliferator-activated receptor-gamma expression in the lung tissue of obese rats. Yonsei Med J 52: 495-501, 2011.

10. Harper JW and Zisman TL: Interaction of obesity and inflammatory bowel disease. World J Gastroenterol 22: 7868-7881, 2016.

11. Nawrocki AR and Scherer PE: The delicate balance between fat and muscle: Adipokines in metabolic disease and musculoskeletal inflammation. Curr Opin Pharmacol 4: 281-289, 2004.

12. Dut R, Dizdar EA, Birben E, Sackesen C, Soyer OU, Besler T and Kalayci O: Oxidative stress and its determinants in the airways of children with asthma. Allergy 63: 1605-1609, 2008.

13. Keaney JF, Jr., Larson MG, Vasan RS, Wilson PW, Lipinska I, Corey D, Massaro JM, Sutherland P, Vita JA and Benjamin EJ, Framingham Study: Obesity and systemic oxidative stress: Clinical correlates of oxidative stress in the framingham study. Arterioscler Thromb Vasc Biol 23: 434-439, 2003.

14. Ota C,Baarsma HA, WagnerDE, Hilgendorff A and Konigshoff M: Linking bronchopulmonary dysplasia to adult chronic lung diseases: Role of WNT signaling. Mol Cell Pediatr 3: 34, 2016.

15. Cui G, Shan L, Hung M, Lei S, Choi I, Zhang Z, Yu P, Hoi P, Wang Y and Lee SM: A novel Danshensu derivative confers cardioprotection via PI3K/Akt and Nrf2 pathways. Int J Cardiol 168: 1349-1359, 2013.

16. Lo SC and Hannink M: PGAM5 tethers a ternary complex containing Keap1 and Nrf2 to mitochondria. Exp Cell Res 314: 1789-1803, 2008.

17. Wicinski M, Malinowski B, Weclewicz MM, Grzesk E and Grzesk G: Anti-atherogenic properties of resveratrol: 4-week resveratrol administration associated with serum concentrations of SIRT1, adiponectin, S100A8/A9 and VSMCs contractility in a rat model. Exp Ther Med 13: 2071-2078, 2017. 
18. Aguirre L, Fernandez-Quintela A, Arias N and Portillo MP Resveratrol: Anti-obesity mechanisms of action. Molecules 19: 18632-18655, 2014.

19. Carpene C, Gomez-Zorita S, Deleruyelle S and Carpene MA Novel strategies for preventing diabetes and obesity complications with natural polyphenols. Curr Med Chem 22: 150-164, 2015.

20. Bayne K: Revised guide for the care and use of laboratory animals available. American phaysiological society. Physiologist 39: 208-211, 1996

21. Aslani MR, Keyhanmanesh R, Khamaneh AM, Ebrahimi Saadatlou MA, Mesgari Abbasi M and Alipour MR: Lung altered expression of il-1 $\beta$ mrna and its signaling pathway molecules in obese-asthmatic male wistar rats. Iran J Allergy Asthma Immunol 15: 183-197, 2016.

22. Hariri $\mathrm{N}$ and Thibault L: High-fat diet-induced obesity in animal models. Nutr Res Rev 23: 270-299, 2010.

23. Simson EL and Gold RM: The lee obesity index vindicated? Physiol Behav 29: 371-376, 1982.

24. Friedewald WT, Levy RI and Fredrickson DS: Estimation of the concentration of low-density lipoprotein cholesterol in plasma, without use of the preparative ultracentrifuge. Clin Chem 18: 499-502, 1972 .

25. Shore SA: Obesity and asthma: Lessons from animal models. J Appl Physiol (1985) 102: 516-528, 2007.

26. Alshammari G, Khan R, Brameld J, Amer S and Lomax MA Gene expression of inflammatory markers in adipose tissue between obese women with polycystic ovary and normal obese women. Eur Rev Med Pharmacol Sci 21: 1099-1105, 2017.

27. Tsuchiya K, Siddiqui S, Risse PA, Hirota N and Martin JG: The presence of LPS in OVA inhalations affects airway inflammation and AHR but not remodeling in a rodent model of asthma. Am J Physiol Lung Cell Mol Physiol 303: L54-L63, 2012.

28. Turner RS, Thomas RG, Craft S, van Dyck CH, Mintzer J, Reynolds BA, Brewer JB, Rissman RA, Raman R and Aisen PS, Alzheimer's Disease Cooperative Study: A randomized, double-blind, placebo-controlled trial of resveratrol for Alzheimer disease. Neurology 85: 1383-1391, 2015.

29. Raj P, Zieroth S and Netticadan T: An overview of the efficacy of resveratrol in the management of ischemic heart disease. Ann N Y Acad Sci 1348: 55-67, 2015

30. Ferretta A, Gaballo A, Tanzarella P, Piccoli C, Capitanio N, Nico B, Annese T, Di Paola M, Dell'aquila C, De Mari M, et al: Effect of resveratrol on mitochondrial function: Implications in parkin-associated familiar Parkinson's disease. Biochim Biophys Acta 1842: 902-915, 2014

31. Chang CC, Lin KY, Peng KY, Day YJ and Hung LM: Resveratrol exerts anti-obesity effects in high-fat diet obese mice and displays differential dosage effects on cytotoxicity, differentiation, and lipolysis in 3T3-L1 cells. Endocr J 63: 169-178, 2016.

32. Chen J, Zhou H, Wang J, Zhang B, Liu F, Huang J, Li J, Lin J, Bai J and Liu R: Therapeutic effects of resveratrol in a mouse model of HDM-induced allergic asthma. Int Immunopharmacol 25: 43-48, 2015.

33. Chen S, Zhao X, Ran L, Wan J, Wang X, Qin Y, Shu F, Gao Y, Yuan L, Zhang Q and Mi M: Resveratrol improves insulin resistance, glucose and lipid metabolism in patients with non-alcoholic fatty liver disease: A randomized controlled trial. Dig Liver Dis 47: 226-232, 2015.

34. Xie HC, Han HP, Chen Z and He JP: A study on the effect of resveratrol on lipid metabolism in hyperlipidemic mice. Afr J Tradit Complement Altern Med 11: 209-212, 2013.
35. Cochius-den Otter SC, Joosten KF, de Jongste JC, Hop WC, de Hoog $\mathrm{M}$ and Buysse CM: Insulin therapy in hyperglycemic children with severe acute asthma. J Asthma 52: 681-686, 2015.

36. Lugogo NL, Bappanad D and Kraft M: Obesity, metabolic dysregulation and oxidative stress in asthma. Biochim Biophys Acta 1810: 1120-1126, 2011.

37. Steffes MW, Gross MD, Lee DH, Schreiner PJ and Jacobs DR, Jr: Adiponectin, visceral fat, oxidative stress, and early macrovascular disease: The coronary artery risk development in young adults study. Obesity (Silver Spring) 14: 319-326, 2006.

38. Ochs-Balcom HM, Grant BJ, Muti P, Sempos CT, Freudenheim JL, Browne RW, McCann SE, Trevisan M, Cassano PA, Iacoviello L and Schunemann HJ: Antioxidants, oxidative stress, and pulmonary function in individuals diagnosed with asthma or COPD. Eur J Clin Nutr 60: 991-999, 2006.

39. Guo R, Li W, Liu B, Li S, Zhang B and Xu Y: Resveratrol protects vascular smooth muscle cells against high glucose-induced oxidative stress and cell proliferation in vitro. Med Sci Monit Basic Res 20: 82-92, 2014.

40. Chen WM, Shaw LH, Chang PJ, Tung SY, Chang TS, Shen CH, Hsieh YY and Wei KL: Hepatoprotective effect of resveratrol against ethanol-induced oxidative stress through induction of superoxide dismutase in vivo and in vitro. Exp Ther Med 11: $1231-1238,2016$

41. Sood A, Qualls C, Arynchyn A, Beckett WS, Gross MD, Steffes MW, Smith LJ, Holvoet P, Thyagarajan B and Jacobs DR, Jr: Obesity-asthma association: Is it explained by systemic oxidant stress? Chest 136: 1055-1062, 2009.

42. Wang T, Liang X, Abeysekera IR, Iqbal U, Duan Q, Naha G, Lin L and Yao X: Activation of the Nrf2-Keap 1 pathway in short-term iodide excess in thyroid in rats. Oxid Med Cell Longev 2017: 4383652, 2017.

43. Wang Z, Ka SO, Lee Y, Park BH and Bae EJ: Butein induction of HO-1 by p38 MAPK/Nrf2 pathway in adipocytes attenuates high-fat diet induced adipose hypertrophy in mice. Eur $\mathbf{J}$ Pharmacol 799: 201-210, 2017

44. Li YJ, Kawada T and Azuma A: Nrf2 is a protective factor against oxidative stresses induced by diesel exhaust particle in allergic asthma. Oxid Med Cell Longev 2013: 323607, 2013.

45. Liu H, Wu HY, Wang WY, Zhao ZL, Liu XY and Wang LY: Regulation of miR-92a on vascular endothelial aging via mediating Nrf2-KEAP1-ARE signal pathway. Eur Rev Med Pharmacol Sci 21: 2734-2742, 2017.

46. Yang X, Wang D, Ma Y, Xu X, Zhu Z, Wang X, Deng H, Li C, Chen M, Tong J, et al: Continuous activation of Nrf2 and its target antioxidant enzymes leads to arsenite-induced malignant transformation of human bronchial epithelial cells. Toxicol Appl Pharmacol 289: 231-239, 2015.

47. Andre DM, Calixto MC, Sollon C, Alexandre EC, Leiria LO, Tobar N, Anhe GF and Antunes E: Therapy with resveratrol attenuates obesity-associated allergic airway inflammation in mice. Int Immunopharmacol 38: 298-305, 2016.

48. Chen B, Lu Y, Chen Y and Cheng J: The role of Nrf2 in oxidative stress-induced endothelial injuries. J Endocrinol 225: R83-R99, 2015.

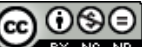

This work is licensed under a Creative Common Attribution-NonCommercial-NoDerivatives 4.0 International (CC BY-NC-ND 4.0) License. 